\title{
PRECIOS HEDÓNICOS: VALORACIÓN DE BIENES PÚBLICOS
}

\author{
HEDONIC PRICES: VALUATION OF PUBLIC GOODS
}

\author{
Christian Rosero ${ }^{1}$, Ronald Campoverde Aguirre ${ }^{2}$
}

Palabras clave:
Bienes públicos;
viviendas; agua;
energía;
características del
vecindario;
suministro de
viviendas y
mercados.

Keywords:

Public goods; housing; water; energy; neighborhood characteristics; housing supply and markets.

\begin{abstract}
Resumen
En la presente investigación se buscó estimar el valor de los bienes públicos a través del precio de las viviendas, en razón de que aún no hay un mercado que pueda valorarlos de forma directa. Se utilizó la metodología de precios hedónicos, con la información del catastro, en el que se destacan atributos físicos, del vecindario, y la inclusión de los servicios públicos. El valor promedio para el agua potable fue de 27.66, para el alcantarillado pluvial de 19.25, 1.26 para el alcantarillado sanitario, 12.48 para el aseo público, 2.34 para la recolección de basura y de 19.35 para el alumbrado.
\end{abstract}

Códigos JEL: C51, H41, R31, R21

\begin{abstract}
In the research, the price of public goods measured through the price of homes was estimated, because they do not have a market that can value them directly. The methodology of hedonic prices was used, with the information from the cadastre of a city of Ecuador, which highlights physical attributes, the neighborhood, and the inclusion of public services. The average value for drinking water was 27.66 , the rainwater drainage of 19.25 , of 1.26 the sanitary sewer, of 12.48 the public toilet, 2.34 for the garbage collection and of 19.35 that of the lighting.
\end{abstract}

JEL Codes: C51, H41, R31, R21

\footnotetext{
${ }^{1}$ Christian Rosero Barzola, Ph.D., Universidad Tecnológica ECOTEC, Posgrado, Km 13.5 Vía Principal Samborondon 092302, Samborondon, Ecuador, Email: crosero@ecotec.edu.ec

${ }^{2}$ Ronald Campoverde Aguirre, Ph.D., Escuela Superior Politécnica del Litoral, ESPOL, Facultad de Ciencias Sociales y Humanísticas, Campus Gustavo Galindo Km. 30.5 Vía Perimetral, P.O. Box 09-01-5863, Guayaquil, Ecuador. Email: recampov@espol.edu.ec
} 


\section{INTRODUCCIÓN}

Se considera como bienes públicos a aquellos que poseen las características de no rivalidad y no exclusión, y que por su naturaleza deben ser asignados por el sistema público (Stiglitz, 1999). Los mismos permiten el desarrollo de varios sistemas, como el abastecimiento de agua y de energía eléctrica (Singh, 2016). Al no existir un mercado directo mediante el cual se pueda medir el valor de los bienes y servicios públicos, es necesario emplear las características intrínsecas del mercado de la vivienda, a través de la Metodología de Precios Hedónicos (MPH), una herramienta aplicada en la ciencia económica para conocer y considerar las preferencias de los consumidores ( $\mathrm{Gu} \& \mathrm{Xu}, 2017$ ).

En estudios previos, diversos autores posicionaron los atributos de la vivienda dentro de cuatro categorías principales: (a) vivienda (Yang, 2001; Ogwang \& Wang, 2003; Revollo, 2009; Falzon \& Lanzon, 2013; Herath \& Maier, 2013; Arce \& Saetama, 2014; Famuyiwa \& Kayode, 2014; Thanasi, 2016; Cadena, Hernández, \& Fuentes, 2016; Romero \& Vargas, 2016; Waltl, 2016; Zambrano-Monserrate, 2016), ambiental (Revollo, 2009; Cadena et al., 2016; Romero \& Vargas, 2016; Zambrano-Monserrate, 2016), (c) vecindario (Yang, 2001; Ogwang \& Wang, 2003; Revollo, 2009; Falzon \& Lanzon, 2013; Herath \& Maier, 2013; Arce \& Saetama, 2014; Famuyiwa \& Kayode, 2014; Thanasi, 2016; Cadena et al., 2016; Romero \& Vargas, 2016; Zambrano-Monserrate, 2016), y (d) bienes públicos (Arce \& Saetama, 2014; Famuyiwa \& Kayode, 2014; Cadena et al., 2016; ZambranoMonserrate, 2016).

La investigación tiene como principal objetivo estimar el valor de los bienes públicos: agua potable, alcantarillado pluvial, alcantarillado sanitario, aseo público, recolección de basura y alumbrado; a través de la Metodología de Precios Hedónicos basado en el mercado de viviendas. Se utilizó el catastro actualizado al 2017 del Gobierno Autónomo Descentralizado del cantón Samborondón para la estimación del modelo, una vez revisada la base se validaron 9,600 casos de avalúo de viviendas en metros cuadrados; adicionalmente se determinó cuáles son las variables más influyentes dentro de la estimación.

En el estudio se presentaron las características que definen a los bienes públicos, el origen y antecedentes de la MPH, así como los atributos que generalmente constituyen a una vivienda. Se establecieron varias de las aplicaciones de la metodología, sus ventajas, limitaciones y los supuestos desarrollados por varios autores. Además, el desarrollo del modelo con base en estudios destacados y una revisión de las variables $\mathrm{y}$ resultados de investigaciones previas sobre precios hedónicos.

\section{METODOLOGÍA}

\section{Precios Hedónicos}

Permite responder a las preguntas: ¿dónde, cuándo y cómo se hizo la investigación?, el autor debe describir de forma clara el diseño de la investigación, los procedimientos utilizados, la unidad de análisis o las medidas de las variables, el diseño muestral, las fuentes de datos y el análisis estadístico. La mayor parte de esta sección debe ser escrita en tiempo pasado.

Los materiales y métodos deben aportar información suficiente para permitir que el trabajo sea replicado. Por ello, se sugiere que el autor comparta el software, código, modelos, algoritmos, protocolos, métodos y otros materiales útiles relacionados con el proyecto.

Stiglitz (1999) argumentó que los bienes públicos presentan dos características fundamentales, son no rivales y no excluyentes. La no rivalidad de un bien se da cuando a pesar de que una persona adicional disfrute del bien, esto no perjudica la utilización del bien por otra persona o grupo de personas (Arce \& Saetama, 2014). Mientras que la no exclusión se refiere a que no es posible negar el acceso o disfrute del bien a ninguna persona o grupo de personas (Pérez, 2016).

Hart y Cowhey (1977) agregaron dos criterios nuevos a los ya establecidos: el primero, la indivisibilidad de los beneficios que ocurre debido a las externalidades en el consumo cuando 
se afecta la utilidad de una persona por el consumo o utilidad de otra; y la segunda, la imposibilidad de apropiación ya que se limita el establecimiento de derechos de propiedad sobre el bien público debido a la presentación de costos muy superiores a los beneficios. En su conjunto, estas características dificultan la provisión de los bienes públicos por parte del mercado y ocasionan que sea el Estado el encargado, manteniendo la legitimidad del suministro (Aktan, 2016). Al no existir un mercado principal, se genera una falla de mercado que imposibilita la valoración de dichos bienes a través de la relación entre oferta y demanda, lo que conlleva a la utilización de metodologías de valoración económica (Pérez, 2016).

Existen distintas metodologías de valoración económica de bienes y servicios, y cada una varía dependiendo de la finalidad para la que se utiliza el bien (Rosero, 2015). Sin embargo, Lavín, Vásquez, Cerda y Orrego (2018) consideró como las más empleadas a: (a) Metodología de Valoración Contingente, (b) Metodología del Costo del Viaje, y (c) Metodología de Precios Hedónicos. La técnica econométrica MPH se emplea para el ajuste de precios en diversos sectores, como el tecnológico, agrícola, automotriz e inmobiliario (Abidoye \& Chan, 2017; Joshi, Ali, \& Berrens, 2017; Moresino, 2019). Azqueta (1998) indicó que el precio de todo bien heterogéneo puede descomponerse, lo que sería equivalente a destinar cierto precio implícito a cada atributo del bien.

La metodología tiene sus inicios en la investigación realizada por Court (1939) para la asociación de fabricantes del sector automotriz, cuando el gobierno estadounidense se preocupó por supuestos oligopolios en Detroit, él explicó que el incremento de los precios de los automóviles se debió a que se mejoró la calidad y características de dichos bienes. Dewey y DeTuro (1950) y Beaty (1952) realizaron los primeros estudios que relacionaron a la MPH con el comportamiento del sector inmobiliario, se enfocaron en demostrar su importancia y la gran inversión producida gracias a las rentas. Lancaster (1966) desarrolló su trabajo entorno a lo que denominó como una "nueva aproximación a la teoría del consumidor" (pág. 133), señalando que la utilidad de los bienes se derivaba de las características del mismo, rechazando la creencia de que la utilidad era implícita.

El enfoque de Lancaster (1966) se fundamentó en tres ideas: (a) los bienes poseen características que determinan su utilidad; (b) cada bien está determinado por más de una característica y generalmente varias características son compartidas por un grupo de bienes; y (c) varias de las características que forman parte de un grupo de bienes también debían estar presentes en los bienes por separado. Rosen (1974) elaboró los principios teóricos para utilizarla dentro de la teoría económica neoclásica y es considerado como el pionero en darle un tratamiento formal a la metodología. Es entonces que en la década de los 70's se le brindó mayor importancia a la MPH, sustentando que establece una interacción entre las características del bien y su precio de venta (Ma, 2019).

French y Lafferty (1984) añadieron los factores externos a la vivienda como variables en el modelo, considerando aspectos como la cercanía a un parque. Dubin y Goodman (1982) sustentaron lo anterior, añadiendo que dichas características son generalmente introducidas como variables binarias. Hasta la actualidad, se aprecia en gran medida su utilización para determinar el precio de viviendas, el cual dependerá de características estructurales (número de habitaciones, tamaño, número de garajes), ambientales (vista al mar, nivel de ruido, cercanía a parques), del vecindario (centros recreativos, nivel de seguridad), y de los servicios públicos (alcantarillado pluvial, agua potable, teléfono) (Pérez, 2016; Rodríguez, Delgado, \& Botello, 2018).

Al emplear la MPH se obtiene como principales beneficios una estimación de valores con base en elecciones efectivas, versatilidad en el uso y que todos los datos utilizados están disponibles para estudio (Azmi \& Ismail, 2017). Ellul, Darmanin y Borg (2019) presentó varias limitaciones o desventajas, como el hecho de que se basa en que los consumidores tienen la posibilidad de elegir la combinación de atributos que deseen en la vivienda dependiendo de su 
precio, lo cual resulta complejo en el mundo real. Mäler (1974) y Brookshire, Thayer, Schulze, y d'Arge (1982) introdujeron cinco supuestos: (a) el mercado es competitivo, es decir que existe perfecta información; (b) se logra la máxima utilidad del consumidor y máximos beneficios para el productor, bajo restricción presupuestaria; (c) el precio de mercado del bien será resultado del conjunto de atributos que posee; (d) posibilidad de fundamentar el valor de un atributo en la demanda del bien, cuando el atributo genera placer al adquirir el bien; y (e) existe movilidad de los agentes en el mercado de estudio.

\section{Descripción de la Metodología}

La función de precio hedónico que se empleó para determinar el precio de la vivienda del cantón Samborondón se formó con tres vectores, el primero contiene las características estructurales $\left(S_{v}=S_{v 1}, S_{v 2}, \ldots, S_{v m}\right)$, el segundo a las características externas o del vecindario $\left(N_{v}=\right.$ $\left.N_{v 1}, N_{v 2}, \ldots, N_{v p}\right)$, y el último se forma a partir de los servicios públicos $\left(G_{v}=G_{v 1}, G_{v 2}, \ldots, G_{v p}\right)$, lo cual se evidencia en la Ecuación 1 a continuación.

$$
P v=f\left(S_{v}, N_{v}, G_{v}\right)
$$

El modelo propuesto se desarrolló para verificar las siguientes hipótesis, basadas en los servicios públicos empleados:

$H_{1}$ : el no tener agua potable influye en el precio de

la vivienda

$\mathrm{H}_{2}$ : el no tener alcantarillado pluvial influye en el precio de la vivienda

$\mathrm{H}_{3}$ : el no tener alcantarillado sanitario influye en el precio de la vivienda

$H_{4}$ : el no tener aseo público influye en el precio de la vivienda

$H_{5}$ : el no tener recolección de basura influye en el precio de la vivienda

$H_{6}$ : el no tener alumbrado influye en el precio de la vivienda

La investigación es positivista, basada en el método deductivo con enfoque cuantitativo. Se realizó un análisis de regresión por mínimos cuadrados ordinarios (MCO), empleando el programa estadístico SPSS. Los datos utilizados se obtuvieron del Catastro del cantón Samborondón, provincia del Guayas del Ecuador, actualizado al 2017. El total de registros original fue de 12,435; luego de validar la base y eliminar valores vacíos se obtuvo un total de 9,600 registros, que representan el $\pm 1 \%$ de margen de error, asumiendo una confianza del $95 \%$ y la máxima dispersión. La base se constituyó con 15 variables, siendo la variable dependiente el avalúo municipal; 13 de las 14 variables regresoras se registraron como binarias, lo cual se muestra en la Tabla 1, Apéndice.

\section{RESULTADOS Y DISCUSIÓN}

La población de Samborondón es de 67,590 habitantes (1.9\% de la población de Guayas), y está dividida entre la zona urbana con un $63.1 \%$ y la rural con $36.9 \%$; la tasa de crecimiento intercensal fue de $4.40 \%$, y la edad media de la población alcanzó los 30 años. La principal actividad desarrollada fue la agricultura, ganadería, silvicultura y pesca, seguida por el comercio al por mayor y menor; generándose durante el año 2010 una recaudación de impuesto a la renta superior a 13 mil dólares, sin embargo, la pobreza aún estaba presente en el 51.9\% de la población. Con respecto a los servicios básicos, se incluye la energía eléctrica, agua, y eliminación de basura, los cuales estaban cubiertos en el 48.5\% de las viviendas; dichos bienes públicos fueron considerados en la estimación realizada con relación al avalúo municipal como variable dependiente y en la Tabla 2, Apéndice se presenta la frecuencia relativa de las variables binarias utilizadas en el modelo (Instituto Nacional de Estadística y Censo [INEC], 2010).

La mayoría de las viviendas son de tipo residencial, ya que solo el $7.2 \%$ constan como arrendadas. Se evidenció un alto nivel de infraestructura, al ser $93.7 \%$ de las viviendas edificadas; $\mathrm{y}$, el $16 \%$ del total son viviendas esquineras. Alrededor del $90 \%$ de ellas se encuentran niveladas, pero exclusivamente el $45.6 \%$ posee camino asfáltico y el $8.6 \%$ aún posee camino de tierra. Además, un $87.6 \%$ registró presencia de bordillos. Se comprobó que un alto porcentaje de viviendas poseen red de agua potable $(99.5 \%)$, alcantarillado sanitario $(98.9 \%)$, recolección de basura $(98.8 \%)$, alumbrado 
(94.7\%) y alcantarillado pluvial (85.6\%); mientras que el aseo público está limitado para dos tercios de la zona (67.5\%). En la estimación del modelo por MCO que se presenta en la Tabla 3. Apéndice se obtuvo la siguiente ecuación de regresión:

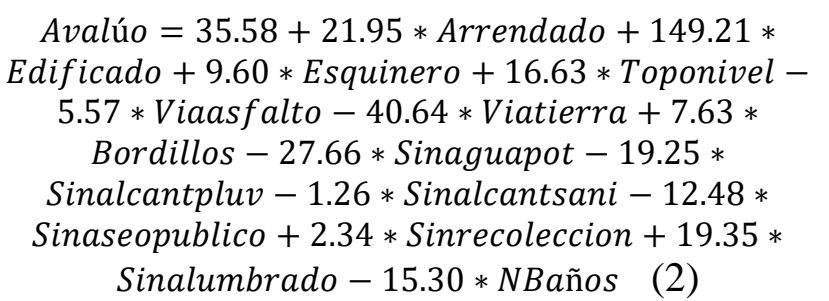

Aplicando la prueba $\mathrm{F}$, se verificó que las variables fueron significativas en conjunto, con un alfa de 0.01. La bondad de ajuste del modelo fue de $48 \%$. El valor promedio del avalúo por metro cuadrado de la vivienda, cuando todas las variables regresoras son igual a cero, resultó ser de \$35.58. Para realizar el análisis de la significancia individual de las variables, sus coeficientes o valores promedio y el efecto que generan varias de ellas sobre el avalúo, se formaron dos grupos.

Para el primer grupo, conformado por las características estructurales y del vecindario, se demostró estadísticamente su significancia individual con un alfa de 0.01. Los valores promedio obtenidos que indican en cuánto varía el avalúo de la vivienda fueron los siguientes: (a) incremento en \$21.95 cuando es arrendada; (b) incremento en $\$ 149.21$ cuando es edificada; (c) incremento en $\$ 9.60$ cuando es esquinera; (d) incremento en $\$ 16.63$ cuando está nivelada; (e) reducción en $\$ 5.57$ cuando posee vía de acceso de asfalto; (f) reducción en $\$ 40.64$ cuando posee vía de acceso de tierra; $(\mathrm{g})$ incremento en $\$ 7.63$ cuando posee bordillos y (h) por cada baño añadido, el precio se devalúa en $\$ 15.30$; en cada caso, ceteris paribus.

El segundo grupo tomó mayor importancia en el estudio, porque incorporó a los servicios públicos. Al nivel de 0.05 , se demostró estadísticamente la significancia de las variables, a excepción de "Sinalcantsani" y "Sinrecoleccion"; comprobando así las hipótesis relacionadas a "Sinaguapot" $\left(\mathrm{H}_{1}\right)$, "Sinalcantpluv" $\left(\mathrm{H}_{2}\right)$, "Sinaseopublico" $\left(\mathrm{H}_{4}\right)$, y "Sinalumbrado" $\left(\mathrm{H}_{6}\right)$. La variación del precio de la vivienda en relación a estos atributos se indica a continuación: (a) reducción en \$27.66 cuando no posee red de agua potable, (b) reducción en $\$ 19.25$ cuando no posee red de alcantarillado pluvial, (c) reducción en $\$ 1.26$ cuando no posee red de alcantarillado sanitario, (d) reducción en $\$ 12.48$ cuando no posee aseo público (e) incremento en $\$ 2.34$ cuando no posee sistema de recolección de basura, y (f) incremento en \$19.35 cuando no posee alumbrado; cetris paribus en cada caso.

La estimación de los valores dados por la población a los bienes públicos, con un intervalo al 95\% de confianza, resultó de: (a) la red de agua potable varió entre 3.32 y 52.01 dólares, (b) el alcantarillado pluvial obtuvo un valor entre 14.46 y 24.04 dólares, (c) el precio del alcantarillado sanitario varió entre 14.96 y 17.49 dólares, (d) el aseo público fluctuó entre 9.16 y 15.81 dólares, (e) el precio de la recolección de basura estuvo entre 10.61 y 15.30 dólares, y (f) el alumbrado obtuvo un valor entre 11.88 y 26.82 dólares.

\section{CONCLUSIONES}

La MPH por más de siete décadas ha contribuido en la estimación de los precios de bienes que no poseen mercado, principalmente para la valoración de los bienes y servicios públicos. Esta investigación se enfocó en un análisis de los diversos atributos estructurales, del vecindario y los servicios públicos que poseen las viviendas del cantón Samborondón. Se midió el grado de impacto de ellos en el avalúo de la vivienda y la valoración que reciben dichos servicios públicos por parte de la población del sector.

Los valores promedio de los servicios públicos obtenidos fueron los siguientes: "Sinaguapot" con 27.66, "Sinalcantpluv" con 19.25, "Sinalcantsani" con 1.26, "Sinaseopublico" con 12.48, "Sinrecoleccion" con 2.34 y "Sinalumbrado" con 19.35. Se encontró que el agua potable y la red de alcantarillado pluvial generan mayor impacto en el avalúo de la vivienda por metro cuadrado, mientras que la 
recolección de basura y el alumbrado generan efectos adversos. Individualmente los coeficientes de los bienes públicos resultaron significativos al 5\%, a excepción de Sinalcantsani y Sinrecoleccion, comprobando así cuatro de las seis hipótesis $\left(\mathrm{H}_{1}, \mathrm{H}_{2}, \mathrm{H}_{4}\right.$ y $\left.\mathrm{H}_{6}\right)$. Sin embargo, en conjunto el modelo resultó significativo.

Los resultados demuestran fiabilidad al coincidir con investigaciones como las realizadas por Famuyiwa y Kayode (2014) y ZambranoMonserrate (2016), en las cuales los bienes públicos logran incrementar el avalúo de la vivienda, principalmente porque incrementan el bienestar de la población. El estudio contribuyó a la obtención de un valor promedio de bienes que no tienen mercado, con lo cual se beneficia a la generación e implementación de políticas públicas que brinden mayores beneficios. Además, se establece un referente de cuánto se puede cobrar a la sociedad por una mejor asignación de los bienes públicos y por mejorar este tipo de servicios.

Una considerable limitación del estudio fue que no se obtuvieron variables adicionales, como teléfono o electricidad, que posiblemente pudieron haber mejorado el modelo. Por lo que, en estudios futuros se recomienda incluir más variables dentro del tema para comprobar si se mejora la bondad de ajuste y la significancia de todos los coeficientes.

\section{REFERENCIAS}

Abidoye, R. B., \& Chan, A. P. (2017). Critical review of hedonic pricing model application in property price appraisal: A case of Nigeria. International Journal of Sustainable Built Environment , 6(1), 250-259. doi:https://doi.org/10.1016/j.ijsbe.2017.02.007

Aktan, C. C. (2016). Regulatory Public Policies: An Introductory Survey. International Journal of Economics and Finance Studies, 8(2), 273-285.

Arce, M., \& Saetama, T. (2014). Determinantes de los precios de vivienda en la ciudad de Cuenca 2011-2012: Un análisis econométrico basado en la metodología hedónica (tesis de pregrado). Cuenca, Ecuador: Universidad de Cuenca.
Azmi, F. A., \& Ismail, S. (2017). A Paradigm Shift from Contingent Valuation Method to Spatial Hedonic Modelling for Heritage Property Valuation. International Academic Conference on Business and Economics (IACBE) Proceeding . Terengganu: Faculty of Economics and Management Sciences (FESP) Universiti Sultan Zainal Abidin (UniSZA).

Azqueta, D. (1998). Valoración Económica de la Calidad Ambiental. Madrid, España: McGraw Hill.

Beaty, J. (1952). Rental real state often a good investment. GP, 5(6), 93-94.

Brookshire, D., Thayer, M., Schulze, W., \& d'Arge, R. (1982). Valuing public goods: a comparison of survey and hedonic approaches. The American Economic Review, 72(1), 165-177.

Cadena, L. A., Hernández, W. Y., \& Fuentes, H. J. (2016). Impacto de la fragmentación espacial sobre el valor de los inmuebles de uso residencial utilizando la metodología de precios hedónicos, caso de estudio: Mosquera, Cundinamarca. Redes de Ingeniería, 7(2), 170-182. doi:10.14483/udistrital.jour.redes.2016.2.a06

Can, A. (1992). Specification and estimation of hedonic housing price models . Regional Science and Urban Economics, 22(3), 453-474. doi:https://doi.org/10.1016/01660462(92)90039-4

Dewey, L. \&. (1950). Should I invest in real state? Medical Economics, 28(3), 85-93.

Dewey, L., \& DeTuro, P. J. (1950). Should I invest in real estate? Medical economics, 28(3), 85-93.

Dubin, R., \& Goodman, A. (1982). Valuation of education and crime neighborhood characteristics through hedonic housing prices . Population and Environment, 5(3), 166-181. doi:https://doi.org/10.1007/BF01257055

Ellul, R., Darmanin, J., \& Borg, I. (2019). Hedonic house price indices for Malta: A mortgage-based approach . CBM Working 
Papers.

Obtenido

de

http://hdl.handle.net/10419/210830

Famuyiwa, F., \& Kayode, G. (2014). Hedonic values of physical infrastructure in house rentals. Journal of Facilities Management, 12(3), 211230. doi:https://doi.org/10.1108/JFM-05-20130027

Frech, H., \& Lafferty, R. (1984). The effect of the California Coastal Commission on housing prices . Journal of Urban Economics , 16(1), 105-123. doi:https://doi.org/10.1016/0094-

1190(84)90053-6

Gu, G., \& Xu, B. (2017). Estudio de precios hedónicos del mercado inmobiliario basado en el impulso del árbol de regresión. Revista de inteligencia computacional avanzada $e$ informática inteligente , 21(6), 1040-1047. doi:https://doi.org/10.20965/jaciii.2017.p1040

Hart, J., \& Cowhey, P. (1977). Theories of collective goods reexamined. The Western Political Quarterly, 30(3), 351-362. doi:https://doi.org/10.1177/10659129770300030 5

Joshi, J., Ali, M., \& Berrens, R. P. (2017). Valuing farm access to irrigation in Nepal: A hedonic pricing model. Agricultural water management, $\quad 181, \quad 35-46$. doi:https://doi.org/10.1016/j.agwat.2016.11.020

Lancaster, K. (1966). A new approach to consumer theory. Journal of Political Economy, 74(2), 132-157.

Lavín, F., Vásquez, A., Cerda, \& Orrego Suaza, S. (2018). Valoración Económica del Medio Ambiente Obtenido de https://www.researchgate.net/profile/Felipe_Lavi n2/publication/332720643_Valoracion_Economi ca_del_Medio_Ambiente/links/5cc62f65a6fdcc 1 d49b75f8e/Valoracion-Economica-del-MedioAmbiente.pdf

Ma, L. (2019). Learning in a hedonic framework: Valuing brownfield remediation . International Economic Review , 60(3), 1355-1387. doi:https://doi.org/10.1111/iere.12389
Mäler, K. (1974). Environmental Economics: A theoretical inquiry. Baltimore, Estados Unidos: Johns Hopkins University Press.

Moresino, F. (2019). A hedonic approach to estimate the price of reliability, energy efficiency and safety for new cars in Switzerland. American Journal of Industrial and Business Management, 9(3), 468-481. doi:https://doi.org/10.4236/ajibm.2019.93031

Pérez, F. (2016). Medio ambiente, bienes ambientales y métodos de valoración . Equidad y Desarrollo(25), 119-158. doi:https://doi.org/10.19052/ed.3725

Rodríguez, J., Delgado, P., \& Botello, T. (2018). Determinantes del precio de la vivienda en Bucaramanga . Equidad y Desarrollo(30), 39-59. doi:https://doi.org/10.19052/ed.3649

Romero, J., \& Vargas, J. (2016). Valoración ambiental de las zonas verdes de una urbanización en Bogotá, Colombia, con el método de precios hedónicos . Semestre Económico, 19(39), 13-30. doi:https://doi.org/10.22395/seec.v19n39a1

Rosen, S. (1974). Hedonic prices and implicit markets: product differentiation in pure competition . Journal of Political Economy, 82(1), 34-55. doi:https://doi.org/10.1086/260169

Rosero, C. (2015). Valoración Económica de Bienes y Servicios Ambientales. Samborondón, Ecuador: Universidad Espíritu Santo .

Singh, J. (2016). Quality of public goods, public policy and human development: A state-wise analysis . Indian Journal of Human Development, 10(2), 215-235. doi:https://doi.org/10.1177/0973703016654537

Stiglitz, J. (1999). Knowledge as a global public good. En I. Kaul, I. Grunberg, \& M. Stern, Global public goods: international cooperation in the 21 st century (págs. 308-325). Washington D.C. , Estados Unidos: Oxford University Press.

Thanasi, M. (2016). Hedonic appraisal of apartments in Tirana. International Journal of Housing Markets and Analysis, 9(2), 239-255. 
doi:https://doi.org/10.1108/IJHMA-03-2015-

0016

Waltl, S. (2016). A hedonic house price index in continuous time. International Journal of Housing Markets and Analysis, 9(4), 648-670. doi:https://doi.org/10.1108/IJHMA-10-2015-

0066
Zambrano-Monserrate, M. (2016). Formación de los precios de alquiler de viviendas en Machala (Ecuador): análisis mediante el método de precios hedónicos . Cuadernos de Economía , 39(109), 12-22.

doi:https://doi.org/10.1016/j.cesjef.2015.10.002

\section{APÉNDICES}

TABLA 1

Descripción de las variables empleadas en el estudio

\begin{tabular}{|c|c|}
\hline Variable & Descripción \\
\hline Avalúo & Variable escalar que representa el precio de la vivienda por metro cuadrado \\
\hline Arrendado & Variable binaria que toma el valor de 1 si la vivienda es arrendada \\
\hline Edificado & Variable binaria que toma el valor de 1 si la vivienda está edificada \\
\hline Esquinero & Variable binaria que toma el valor de 1 si la vivienda es esquinera \\
\hline Toponivel & Variable binaria que toma el valor de 1 si la vivienda está nivelada \\
\hline Viaasfalto & Variable binaria que toma el valor de 1 si la vivienda posee vía de acceso de asfalto \\
\hline Viatierra & Variable binaria que toma el valor de 1 si la vivienda posee vía de acceso de tierra \\
\hline Bordillos & Variable binaria que toma el valor de 1 si la vivienda posee bordillos \\
\hline Sinaguapot & Variable binaria que toma el valor de 0 si la vivienda posee red de agua potable (Bien público) \\
\hline Sinalcantpluv & $\begin{array}{l}\text { Variable binaria que toma el valor de } 0 \text { si la vivienda posee red de alcantarillado pluvial (Bien } \\
\text { público) }\end{array}$ \\
\hline Sinalcantsani & $\begin{array}{l}\text { Variable binaria que toma el valor de } 0 \text { si la vivienda posee red de alcantarillado sanitario (Bien } \\
\text { público) }\end{array}$ \\
\hline Sinaseopublico & Variable binaria que toma el valor de 0 si la vivienda posee servicio de aseo público (Bien público) \\
\hline Sinrecoleccion & $\begin{array}{l}\text { Variable binaria que toma el valor de } 0 \text { si la vivienda posee servicio de recolección de basura (Bien } \\
\text { público) }\end{array}$ \\
\hline Sinalumbrado & Variable binaria que toma el valor de 0 si la vivienda posee alumbrado (Bien público) \\
\hline NBaños & Variable escalar que indica el número de baños en la vivienda \\
\hline
\end{tabular}

Fuente: Elaboración propia 
TABLA 2

Frecuencia relativa de las variables utilizadas

\begin{tabular}{|c|c|c|}
\hline Variable & $\mathbf{S i}(\mathbf{1})$ & No $(0)$ \\
\hline Arrendado & 7.2 & 92.8 \\
\hline Edificado & 93.2 & 6.3 \\
\hline Esquinero & 16 & 84 \\
\hline A nivel & 89.1 & 10.9 \\
\hline Vía de asfalto & 45.6 & 54.4 \\
\hline Vía de tierra & 8.6 & 91.4 \\
\hline Bordillos & 87.6 & 12.4 \\
\hline Agua potable & 99.5 & .5 \\
\hline Alcantarillado pluvial & 85.6 & 14.4 \\
\hline Alcantarillado sanitario & 98.9 & 1.1 \\
\hline Aseo público & 67.5 & 32.5 \\
\hline Recolección de basura & 98.8 & 1.2 \\
\hline Alumbrado & 94.7 & 5.3 \\
\hline
\end{tabular}

Fuente: Elaboración Propia

TABLA 3

Modelo de precios hedónicos

\begin{tabular}{lrrrrrr}
\hline \multicolumn{1}{r}{ Modelo } & Beta & Error Est. & $\mathbf{t}$ & Valor p & \multicolumn{2}{c}{ Intervalo de Confianza al 95\% } \\
& & & & & Limite Inferior & Limite Superior \\
\hline Constante & 35.586 & 4.674 & 7.614 & $0.000^{* * *}$ & 26.425 & 44.747 \\
Arrendado & 21.954 & 2.627 & 8.357 & $0.000^{* * *}$ & 16.805 & 27.103 \\
Edificado & 149.212 & 3.051 & 48.898 & $0.000^{* * *}$ & 143.230 & 155.193 \\
Esquinero & 9.605 & 1.874 & 5.127 & $0.000^{* * *}$ & 5.933 & 13.278 \\
Toponivel & 16.639 & 2.209 & 7.531 & $0.000^{* * *}$ & 12.308 & 20.970 \\
Viaasfalto & -5.576 & 1.446 & -3.857 & $0.000^{* * *}$ & -8.410 & -2.742 \\
Viatierra & -40.645 & 2.934 & -13.852 & $0.000^{* * *}$ & -46.397 & -34.893 \\
Bordillos & 7.635 & 2.598 & 2.939 & $0.003^{* * *}$ & 2.543 & 12.727 \\
Sinaguapot & -27.667 & 12.420 & -2.228 & $0.026^{* *}$ & -52.012 & -3.322 \\
Sinalcantpluv & -19.255 & 2.446 & -7.872 & $0.000^{* * *}$ & -24.049 & -14.460 \\
Sinalcantsani & -1.266 & 8.280 & -0.153 & 0.878 & -17.497 & 14.965 \\
Sinaseopublico & -12.489 & 1.696 & -7.362 & $0.000^{* * *}$ & -15.815 & -9.164 \\
Sinrecoleccion & 2.344 & 6.610 & 0.355 & 0.723 & -10.614 & 15.301 \\
Sinalumbrado & 19.350 & 3.811 & 5.078 & $0.000^{* * *}$ & 11.881 & 26.820 \\
NBaños & -15.307 & 0.195 & -78.393 & $0.000^{* * *}$ & -15.690 & -14.925 \\
$\mathrm{~F}$ & & & & $0.000^{* * *}$ & & \\
$\mathrm{R}^{2}$ ajustado & 0.481 & & & & & \\
\hline
\end{tabular}

Nota. $\mathrm{N}=9600$ viviendas. $* \mathrm{p}<.10 . * * \mathrm{p}<.05 . * * * \mathrm{p}<.01$.

Fuente: Elaboración propia 